\title{
Zusammenfassende Thesen
}

Klagen gegen den Hunger sind weltweit möglich und angesichts der fortbestehenden globalen Nahrungsunsicherheit, unter der ein Großteil der Weltbevölkerung leidet, nötig. Sie stellen ein Instrument dar, um Diskurse über die Ursachen von chronischer Mangel- und Unterernährung anzuregen, die Ergreifung erforderlicher Überwindungsmaßnahmen zu beschleunigen und unverzichtbare Mindestschritte einzufordern.

\section{Universelles Menschenrecht}

Dass es sich beim Recht auf Nahrung um ein gültiges Menschenrecht handelt, ergibt sich aus seiner ausdrücklichen Kodifizierung in zahlreichen Verfassungsurkunden und völkerrechtlichen Verträgen. Auch anhand der vielfältigen Rechtsprechung sowie regelmäßig und einstimmig verabschiedeter UN-Resolutionen, Weltgipfelerklärungen und der FAO-Leitlinien zur schrittweisen Umsetzung des Rechts auf Nahrung konnte die gewohnheitsrechtliche Geltung des ,right to food“" nachgewiesen werden.

\section{Begründung}

Die Anerkennung des Rechts auf Nahrung als unverzichtbares Menschenrecht lässt sich unterschiedlich begründen. In einer demokratischen und auf dem Prinzip der Menschenwürde gründenden Gesellschaft sollte niemand Hunger leiden, weshalb allen hilfsbedürftigen Menschen ein soziales Zugangsrecht zuzusprechen ist. Aus dem Anspruch des Einzelnen auf sozioökonomische Autonomie folgt zugleich ein Recht auf Schutz und Förderung der eigenständigen Verwirklichung, sei es unmittelbar durch Eigenproduktion von Nahrung oder mittelbar durch freie bzw. abhängige (Lohn-)Arbeit. Generell besteht die kommunikative Funktion des ,right to food" darin, es marginalisierten Menschen, die unter Hunger leiden, zu ermöglichen, die Aufklärung von Hungerursachen und die Einleitung von Überwindungsschritten, wo immer dies notwendig ist, zu verlangen. Auch wenn die Erforschung der Gründe für Nahrungsunsicherheit eine wissen- 
schaftliche Aufgabe ist und die Durchsetzung von wirksamen Anti-Hunger-Programmen eine politische Angelegenheit bleibt, ist das Recht auf Nahrung zumindest ein geeignetes Instrument, um bestehende soziale Exklusionslagen zu skandalisieren, die Gesellschaft auf extreme Missstände aufmerksam zu machen und notwendige wissenschaftliche und politische Diskurse anzuregen, zu beschleunigen und zu reflektieren.

\section{Durchsetzung}

Als Durchsetzungsmechanismen sind neben Gerichtsverfahren quasi-judizielle Individualbeschwerden vor völkerrechtlichen Menschenrechtsausschüssen in Betracht zu ziehen. Mit Blick auf das Recht auf Nahrung hat etwa das Verfahren „Social and Economic Rights Action Center“ (2001) vor der Afrikanischen Menschenrechtskommission internationale Aufmerksamkeit erregt, in dem eine Verletzung von Subsistenzrechten des Ogoni-Volks durch Nigeria in Folge einer rücksichtslosen Ölförderung festgestellt wurde. Im Rahmen des Berichtsverfahrens vor dem UN-Sozialausschuss können strukturelle Fragen des Menschenrechtsschutzes einfacher thematisiert und die Angemessenheit genereller Maßnahmen zur progressiven Verwirklichung des Rechts auf Nahrung begutachtet werden. Die Ausschussempfehlungen sind zwar meist sehr allgemein gehalten, benennen indes systemische Defizite in einem Vertragsstaat und regen einen „konstruktiven Dialog“ an. In einzelnen Abschließenden Bemerkungen stellte das CESCR zudem ausdrücklich Paktverletzungen fest und forderte konkrete Abhilfemaßnahmen. So wurden gewaltsame Vertreibungen von indigenen Völkern oder Kleinproduzierenden vom Komitee regelmäßig eindeutig als Menschenrechtsverletzungen bezeichnet.

\section{Das Verpflichtungspotenzial}

Das Zweistufenmodell, das vom UN-Ausschuss für wirtschaftliche, soziale und kulturelle Rechte entwickelt wurde, steht für ein gesamtheitliches Menschenrechtsverständnis, das dem Recht auf Nahrung (Art. 11 I IPwskR) ein weites Verpflichtungspotenzial zuschreibt. Statt den normativen Gehalt des ,right to food“" aus individueller Perspektive zu entfalten, entwirft der Ausschuss Grundelemente, die auf strukturelle Voraussetzungen der Menschenrechtsgewährleistung verweisen, und benennt korrespondie- 
rende negative und positive Staatenpflichten. Alle Vertragsparteien müssen den Zugang zu angemessener Nahrung achten, schützen und gewährleisten. Ausgehend von den vielfältigen Pflichtentypen, die der UN-Sozialausschuss entwickelt hat, wurden zwei Wege zur Operationalisierung des Rechts auf Nahrung untersucht. Neben der Prozeduralisierung der Ausschussdogmatik durch die Ausformulierung formeller Anforderungen wurde eine Materialisierung durch die Annahme subjektiver Zugangsrechte erwogen.

\section{Prozeduralisierung}

Ausgangspunkt eines prozeduralen Menschenrechtsverständnisses bilden die vom UN-Sozialausschuss beschriebenen Formalpflichten wie die Pflicht zur Erstellung von Strategien, zur Ergreifung gesetzgeberischer Maßnahmen sowie zur Einführung von Rechtsbehelfen. Selbst wenn die weltweiten Ursachen von chronischer Unter- und Mangelernährung zu komplex und mögliche Gegenmaßnahmen zu vielschichtig sind, um aus dem Recht auf Nahrung im Einzelfall den einzig richtigen Weg zur Rechtsverwirklichung abzuleiten, ist von jeder Regierung die Beachtung formeller Gesichtspunkte bei der Durchführung ihrer Anti-Hungerpolitik zu verlangen. Ein menschenrechtsbasierter Ansatz für Nahrungssicherheit zielt auf eine Institutionalisierung der Anti-Hungerpolitik, auf die Beteiligung der Zivilgesellschaft und auf eine ständige Wirksamkeitskontrolle eingeführter Programme. Am Beispiel Brasiliens konnte gezeigt werden, dass die Umsetzung eines ,human rights-based approach to food security“ erfolgversprechend ist. Eine Gesamtstrategie und vielfältige Programme, die von unterschiedlichen Gremien unter Beteiligung zivilgesellschaftlicher Organisationen erarbeitet, umgesetzt und überwacht werden, haben zu einer wesentlichen Intensivierung der Anti-Hungerpolitik beigetragen.

Allerdings wurde die Verwissenschaftlichung, Politisierung und Verrechtlichung der brasilianischen Nahrungssicherheitspolitik nicht durch Gerichtsurteile angeregt, sondern geht auf die Wahl eines neuen Präsidenten zurück, der sich zivilgesellschaftliche Forderungen und Pläne zu eigen machte. Gleichwohl könnte der Angemessenheitstest (reasonableness standard), den das südafrikanische Verfassungsgericht zur Überprüfung von Programmen zur schrittweisen Verwirklichung wirtschaftlicher und sozialer Rechte entwickelt hat, zur Judizialisierung der Formalpflichten genutzt werden. Demnach ist jeder Staat als verpflichtet anzusehen, ange- 
messene Programme zur progressiven Verwirklichung des Rechts auf Nahrung einzuleiten, die sowohl unmittelbar Bedürftige berücksichtigen als auch die Stabilität der Nahrungssicherheit bezwecken.

\section{Materialisierung}

Neben der Beachtung formeller Gesichtspunkte, die menschenrechtliche Standards im Bereich einer guten Anti-Hungerpolitik bezeichnen, stellt die Materialisierung des Rechts auf Nahrung einen geeigneten Ansatz zur Operationalisierung der vom UN-Sozialausschuss entwickelten Pflichtentypen dar. In diesem Sinne verweist die Erklärung kleinbäuerlicher Rechte, die von der transnationalen Bewegung La Via Campesina eingefordert werden, auf die Möglichkeit subjektiver Rechte auf Nahrungssicherheit und Ernährungssouveränität. Ausgehend vom Kerngedanken, dass das Recht auf Nahrung nicht nur die Versorgung von Hilfsbedürftigen, sondern zugleich die Achtung, den Schutz und die Förderung der eigenmächtigen Selbstversorgung bezweckt, wurden drei Zugangsrechte entfaltet.

\section{a. Fürsorgerecht}

Das Recht auf Fürsorge steht bedürftigen Personen zu. Im Wege von Klagen gegen den Hunger ist die Legislative zu verpflichten, den Anspruch auf ein soziokulturelles Minimum für alle, das den Zugang zu Nahrung umfasst, auszugestalten und zu beziffern. Selbst ärmere Staaten wären finanziell in der Lage, einen sozialen Basisschutz zu gewährleisten. Darüber hinaus sollten Menschen, die sich in akuten Notlagen befinden, unmittelbare Unterstützungsansprüche zugesprochen werden. So hat das indische Verfassungsgericht im Jahr 2001 eine Klage im öffentlichen Interesse zum Anlass genommen, um die bestehenden Nahrungssicherheitsprogramme durch einstweilige Anordnungen zu effektivieren und damit Millionen von Menschen Genugtuung zu verschaffen.

\section{b. Das Recht auf Selbstversorgung}

Das Recht auf Selbstversorgung schützt die Herstellung von Nahrung zur Eigennutzung. Kein Staat darf sich an Vertreibungen oder Umweltver- 
schmutzungen beteiligen, die Kleinproduzierenden den Zugang zu natürlichen Ressourcen, die diese zu Subsistenzzwecken nutzen, verunmöglichen. So hat der Internationale Gerichtshof in seinem Gutachten zum Mauerbau (2004) festgestellt, dass eine willkürliche Entziehung landwirtschaftlich genutzter Ressourcen eine Menschenrechtsverletzung darstellen kann. In ländlichen Regionen, die sowohl eine hohe Landkonzentration als auch einen hohen Armutsanteil aufweisen, ist ein Recht auf Agrarreform einzufordern. In diesem Sinne hat der UN-Sozialausschuss in seinen Abschließenden Bemerkungen die Beschleunigung von Landreformen angemahnt, um marginalisierten Gruppen einen Zugang zu produktiven Ressourcen zu ermöglichen.

\section{c. Das Recht auf ein angemessenes Einkommen}

Das Recht auf ein ausreichendes Einkommen zielt mittelbar auf die Sicherung des Nahrungszugangs. Aus der Spruchpraxis des UN-Sozialausschusses ergibt sich, das Lohnuntergrenzen ein geeignetes Mittel sind, um das Recht auf angemessene Löhne (Art. 7 a) ii) IPwskR) zu gewährleisten. Zudem wurde ein Recht auf faire Agrarpreise angedacht, das Kleinproduzierenden einen ausreichenden Lebensstandard sichern soll. Staaten sollten die bäuerliche Landwirtschaft fördern, um zu vermeiden, dass kleinere Agrarbetriebe in wirtschaftliche Notlagen geraten und die betroffenen Haushalte unter Nahrungsunsicherheit leiden.

\section{Transnationale Schutzdimension}

Da der Schutz und die Verwirklichung des Rechts auf Nahrung in Zeiten globalisierter Weltmärkte und Umweltkrisen nicht mehr allein von den Entscheidungen einzelstaatlicher Regierungen abhängt, sollten alle Staaten dieses auch in grenzüberschreitenden Konstellationen achten und schützen. Wäre es den Vertragsstaaten erlaubt, die wsk-Rechte im Ausland zu beeinträchtigen, stünde dies im Widerspruch zu der im UN-Sozialpakt ausdrücklich niedergelegten Kooperationspflicht. Die transnationale Schutzdimension folgt zugleich aus der völkergewohnheitsrechtlichen Geltung des Rechts auf Nahrung. Alle Völkerrechtssubjekte sind verpflichtet, weder den Genuss des Rechts auf Nahrung in Drittstaaten direkt oder mittelbar zu beeinträchtigen noch Maßnahmen zu ergreifen, welche 
die Nahrungssicherheit in anderen Ländern erheblich gefährden. Nach einem zeitgemäßen Verständnis sollte die Anwendbarkeit der Menschenrechte nicht vom Maßstab der effektiven Kontrolle, sondern von den tatsächlichen Einflussmöglichkeiten und der Vorhersehbarkeit von Beeinträchtigungen abhängig gemacht werden. In prozeduraler Hinsicht sind alle Staaten und Internationalen Organisationen als verpflichtet anzusehen, die Auswirkungen ihrer Politik auf das Recht auf Nahrung in anderen Ländern zu evaluieren. Diesem Gebot, die Menschenrechtsverträglichkeit des eigenen Handelns zu prüfen, müssen zugleich transnational agierende Unternehmen nachkommen, die das Nahrungssystem zunehmend dominieren und generell zur Achtung der Menschenrechte anderer verpflichtet sind.

Im Rahmen des Berichtsverfahrens vor dem UN-Sozialausschuss ist es Nicht-Regierungsorganisationen bereits gelungen, extraterritoriale Staatenpflichten zu thematisieren. Die begutachteten Sachverhalte betrafen den Vorwurf des Agrardumpings und der expansiven Förderung von Biokraftstoffen. Anhand der eigenen Fallstudien zu beiden Themen konnte aufgezeigt werden, dass transnationale Gefährdungslagen sowohl durch öffentliche als auch durch private Akteure verursacht werden.

\section{Agrardumping}

Die Gewährung von Exportsubventionen verletzt die grenzüberschreitende Achtungspflicht, soweit sie die Preisentwicklung des Agrarrohstoffs global hemmt und Billigimporte ermöglicht, die lokal Produzierende aus dem Markt drängen. Generell sollten es reichere Länder vermeiden, sich mit Blick auf sensible Agrarerzeugnisse, die genauso gut in den ,am wenigsten entwickelten Ländern“ hergestellt werden könnten, zu Netto-Exporteuren zu entwickeln. Die auf Ebene der Welthandelsorganisation bestehenden Rechtsschutzmöglichkeiten ermöglichen es ärmeren Mitgliedern nur ansatzweise, ihre Landwirtschaft vor Billigimporten zu bewahren.

NGO-Studien zum Bananenhandel verdeutlichen, dass Agrardumping nicht nur auf staatliche Subventionierungen, sondern im Einzelfall auch auf eine hohe Marktkonzentration im Lebensmitteleinzelhandel zurückgeführt werden kann. So wird die Niedrigpreispolitik deutscher Supermarktketten als Grund genannt, weshalb sich die Arbeitsbedingungen in den Anbauländern tropischer Früchte nicht verbessern. Angesichts systematischer Verletzungen arbeitsrechtlicher Schutzstandards und der systematischen Unterwanderung gesetzlicher Mindestpreise sollte der deutsche Ein- 
zelhandel, um seiner Sorgfaltspflicht gegenüber den Menschenrechten anderer gerecht zu werden, erwägen, ausschließlich Bananen zu vermarkten, die den Standards des „fairen Handels“ genügen.

\section{EU-Biokraftstoffpolitik}

Die Europäische Union bleibt den Nachweis, dass die bestehenden Gefahren ihrer Biokraftstoffförderung für das Recht auf Nahrung in anderen Ländern angesichts überwiegend positiver Folgen zu vernachlässigen sind, bis heute schuldig.

Nach der Erneuerbare-Energien-Richtlinie müssen die Mitgliedstaaten bis 2020 sicherstellen, dass mindestens $10 \%$ der im Verkehrssektor verbrauchten Energie aus alternativen Quellen stammen. Die Kommission hat es versäumt, die Auswirkungen dieser Zielsetzung, die zu einer erheblichen Steigerung der Nachfrage nach Biomasse und entsprechend zu erhöhten Importen von Agrarrohstoffen aus Drittstaaten führt, aus menschenrechtlicher Perspektive zu evaluieren. Wissenschaftliche Studien legen nahe, dass die steigende Nutzung von Biokraftstoffen in der EU zu steigenden Weltagrarpreisen beiträgt, insbesondere für pflanzliche Öle, die weltweit zur Zubereitung von Speisen genutzt werden. Zudem belegen NGO-Feldstudien, dass der künstliche Markt für „,biofuels“ in der Union großflächige Landgeschäfte im globalen Süden angeregt hat, die zu einer Verschlechterung der Nahrungssicherheit lokaler Gemeinden geführt haben.

Eine den Menschenrechten verpflichtete internationale Agrarpolitik müsste sich generell für die Etablierung verbindlicher ökologischer und sozialer Nachhaltigkeitsstandards einsetzten, um Konflikte zwischen dem großflächig-industriellen Produktionsmodell und dem Recht auf Nahrung zu entschärfen und damit gleichzeitig die kleinbäuerliche Landwirtschaft zu stärken. 